\title{
Hydraulic characteristics of flow through angled baffle-plates in an open channel
}

\author{
C. H. J. Bong (i) and C. V. Liow \\ Department of Civil Engineering, Faculty of Engineering, Universiti Malaysia Sarawak, Sarawak, Malaysia
}

\begin{abstract}
This paper aims to study the flow characteristics through angled baffle-plates in an open channel. The experiments were conducted in a self-circulating hydraulic flume with three designated baffle-plates' angles $\left(30^{\circ}, 60^{\circ}\right.$, and $\left.90^{\circ}\right)$ and three flow conditions (high, medium, and low). Results showed that all the designed angles for the different flow conditions do retard the flow resulting in reduced downstream mean discharge as compared to without the baffle-plates. The baffle-plates installed at $90^{\circ}$ showed the greatest percentage of mean discharge reduction for all the flow conditions (ranged between $5.32 \%$ and $11.67 \%$ ) as compared to $60^{\circ}$ (ranged between $3.96 \%$ and $8.3 \%$ ) and $30^{\circ}$ (ranged between $3.55 \%$ and $7.07 \%$ ). An equation to predict the relative flow depth increase due to the retarding behaviour of the angled baffle-plates structure was also developed in this study using multiple linear regression. The proposed equation has an $R^{2}$ value of $0.69, R_{\text {adj }}^{2}$ value of 0.62 , Mallow's $C_{p}$ value of 2 and a mean square error (MSE) value of 0.4548 , which is the best among all the possible regression models for this study. The equation can be used by the designer in determining the desired angle for the baffle-plates structure to prevent overflow at upstream of the structure.
\end{abstract}

\section{ARTICLE HISTORY}

Received 26 August 2018

Accepted 21 July 2019

\section{KEYWORDS}

Angled baffle-plates;

detention; flow regulator; mean discharge reduction; relative flow depth increase; stormwater management

\section{Introduction}

The current study presented an experimental study on the hydraulic characteristics and flow retarding behaviour for baffle-plates arranged in a certain angled manner. The angled baffle-plates created a curved flow path and exerted a backwater effect that retards the approaching flows. This can be useful for peak flow reduction in urban stormwater management and managing the conveyance of flows to within acceptable flow rates (Newton et al. 2014). This study focused on how the angle of the baffle-plates influences the flow retardation property and also the backwater effect. The findings of this study extend prior studies on the effects of curved flow to flow resistance. For example, Leopold (1994) reported that the degree of the flow curve angle is related to flow resistance. Besides that, the significance of the curve angle of the flow has been studied, such as in $30^{\circ}, 60^{\circ}, 120^{\circ}$, and $150^{\circ}$ curved open channels. For example, Blanckaert and De Vriend (2005) measured the turbulent stress components of curved currents and analysed the flow turbulence in a sharp $120^{\circ}$ bend. Zhang and Shen (2008) presented a 3-D numerical model to determine water depth changes and longitudinal and transverse velocity profiles in curved channels. Gholami et al. (2016) studied the water depth patterns change in a $120^{\circ}$ sharp bend using the numerical model. Experiments to study the flow features in a strongly curved $90^{\circ}$ and $30^{\circ}$ channel were also reported in Seyedashraf and Akhtari $(2016,2017)$. Apart from that, the study of investigation on louves and angled bar racks demonstrates that the angle parameters such as rack angle and bar angle are strongly interrelated with respect to the head loss (Albayrak et al. 2018). Though these studies gave insights on the flow retarding property of angled flow, there is still a lack of information about the application and the optimum design of angled baffle-plates in getting the required discharge reduction for an open channel.

\section{Dimensional analysis}

To describe flow through a retarding structure, the dominant variables can be described in three components, namely (i) geometric properties of the structure; (ii) effect of structure geometry to flow; and (iii) flow properties (Ubing et al. 2017). Hence, for this study, the variables can be stated as

$$
\theta, N, w, l, y_{0}, y_{1}, q_{0}, B, g, v
$$

where $\theta$ is the angle of the baffle-plates along both sidewalls of the channel (in radian); $N$ is the number of baffle-plates; $w$ is the width of each baffle-plates $(\mathrm{m}) ; l$ is the spacing between baffle-plates (m); $y_{0}$ is the flow depth upstream $(\mathrm{m}) ; y_{1}$ is the flow depth just before the retarding structure $(\mathrm{m}), q_{0}$ is the unit discharge at upstream section $\left(\mathrm{m}^{2} / \mathrm{s}\right) ; B$ is the channel width $(\mathrm{m}) ; g$ is the acceleration of gravity $\left(\mathrm{m} / \mathrm{s}^{2}\right)$; and $v$ is the kinematic viscosity of water $\left(\mathrm{m}^{2} / \mathrm{s}\right)$ (see Figure 1). Gradually varied flow is assumed to exist between sections 0 and 1 (between $y_{0}$ and $y_{1}$ ), where $y_{0}$ indicates uniform flow depth well upstream of the angled baffle-plates structure. However, limited flume length in the current study hampered the measurement of $y_{0}$, and a set of experiment for uniform flow without the angled baffle-plates was carried out to determine the $y_{0}$.

Since in the current study, the effect of baffle-plates angle $\theta$ is the focus of the experiment; the variables $N, w, l, B$ are fixed and can be excluded. The remaining variables can be reduced to four dimensionless parameters through dimensionless analysis:

$$
\theta, \frac{y_{1}}{y_{0}}, \frac{q_{0} / y_{0}}{\sqrt{g y_{0}}}, \frac{q_{0}}{v}
$$

The first term in Equation (2) defines the geometric properties of the structure (in this case, the angle of the baffleplates). The second term defines the effect on the retarding 